\title{
Debatable evidence for the adverse drug reactions to local anaesthetics
}

\author{
Abstracted from \\ Liu W, Yang X, Li C, Mo A. \\ Adverse drug reactions to local anesthetics: a systematic review. \\ Oral Surg Oral Med Oral Pathol Oral Radiol. 2013; 115: 319-327. \\ Address for correspondence: Anchun Mo, No. 14, Third section South Renmin Road Chengdu, \\ 610041 China. E-mail: moanchun@163.com
}

\section{Question: What adverse drug reactions are associated with local anaesthetics?}

Data sources Medline, Embase, Chinese National Knowledge Infrastructure VIP Database for Chinese Technical Periodicals (and Chinese Biomedical Literature Database.

Study selection Randomised controlled trials (RCTs), non-RCTs, case-control studies, case reports, case series and cross-sectional studies that focused on adverse drug reactions of local anaesthetics were considered.

Data extraction and synthesis Data abstraction was conducted independently by two reviewers, and summary data and a metaanalysis presented.

Results One hundred and one studies reporting 1645 events were included. Seven of these were deaths. Lidocaine (43.17\%) and bupivacaine (16.32\%) were the most often involved local anaesthetics. According to the meta-analysis, the risk of using LA alone was lower than when combined with epinephrine.

Conclusions The present study demonstrated that the adverse drug reactions of local anaesthetics could not be ignored, especially in oral and ophthalmologic treatments. Some adverse drug reactions could be avoided by properly evaluating the conditions of patients and correctly applying local anaesthetics.

\section{Commentary}

This article purports to be a systematic review to analyse adverse drug reactions associated with the use of local anaesthetics. It is intended to report on safety issues attached to the use of these drugs. Basically, this paper is a compilation of selected articles reporting on the morbidity and mortality, as well as a whole menu of untoward outcomes attributed to a wide variety of substances, not just anaesthetics alone, but adjunctive substances associated with the delivered medicaments, ie epinephrine, other vaso-active substances, sulfites and preservatives - further confused with a wide variety of combinations.

Immediately, the interested reader is confronted with a confusing defined question. Trying to understand comparisons in a reliable way (as it is often said: comparing 'apples with oranges'), we are lost in trying to generalise outcomes from data of mixed and possibly incongruent studies.
First, the introduction states, 'we could analyze this clinical problem in a logical way', which flies in the face of the confusing and sometimes unrelated data and reportage. The search strategies, aside from the general descriptor noting 'drug reactions' were far too broad in that the moieties compared were so varied, methods of their administration and use unheeded, anaesthetic structural type (molecular structure) not separated, quantities administered (dose) not considered, and routes of administration inconsequential. Therefore, zeroing in on reliable generalisations as to cause and effect was not a fair intervention.

The paper talks of adverse reactions, including untoward events which may or may not be directly drug related, such as side effects, hypersensitivity, allergic reactions, interactions with other medicines that the patient may be taking and/or attributed to other substances not of anaesthetic category. Errors in administration occurred, such as intravascular placement, effects of poor site selection, blame on epinephrine attributed to the anaesthetic itself, and inability to separate cause from mixtures of several drugs.

Only English and Chinese language searches were included, disregarding large tomes of pertinent literature. Even though an attempt was made to eliminate bias in the selection of data, the compilation included was subject to human error and bias. However, the most important anaesthetic related dangerous outcome, comparing anaesthetics with and without epinephrine, as well as other vasoconstrictors was not brought into play. Not only was there uncontrolled and mixed reportage of anaesthetic type, but factors such as psychologic stress, vaso-vagal effects and underlying health issues were poorly addressed.

This meta-analysis and systematic review is a report of different kinds of morbidity and mortality associated with the administration of a large variety of local anaesthetic products, but does not sufficiently separate, delineate and categorise appropriate causation. This blanket report only alerts the clinician that there are multiple possible reasons for an adverse event due to local anaesthetic use.

Ronald I. Maitland New York University College of Dentistry, New York, USA

Evidence-Based Dentistry (2013) 14, 51. doi:10.1038/sj.ebd.6400935 